\title{
The onset of star formation in primordial haloes
}

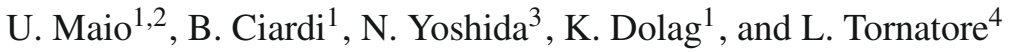 \\ 1 Max-Planck-Institut fuer Astrophysik, Karl-Schwarzschild-Straße 1, 85748 Garching bei München, Germany \\ e-mail: maio@mpa-garching.mpg.de \\ 2 Max-Planck-Institut fuer extraterrestrische physik, Giessenbachstraße 1, 85748 Garching bei München, Germany \\ e-mail: umaio@mpe.mpg.de \\ 3 IPMU, U-Tokyo 5-1-5 Kashiwanoha, Kashiwa, Chiba 277-8568, Japan \\ 4 INAF - Osservatorio astronomico di Trieste, via Tiepolo 11, 34143 Trieste, Italy
}

Received 30 March 2009 / Accepted 26 May 2009

\section{ABSTRACT}

\begin{abstract}
Context. Star formation remains an unsolved problem in astrophysics. Numerical studies of large-scale structure simulations cannot resolve the process and their approach usually assumes that only gas denser than a typical threshold can host and form stars. Aims. We investigate the onset of cosmological star formation and compare several very-high-resolution, three-dimensional, $\mathrm{N}$-body/SPH simulations that include non-equilibrium, atomic and molecular chemistry, star formation prescriptions, and feedback effects.

Methods. We study how primordial star formation depends on gas density threshold, cosmological parameters, and initial set-ups. Results. For mean-density initial conditions, we find that standard low-density star-formation threshold $\left(0.2 \mathrm{~h}^{2} \mathrm{~cm}^{-3}\right)$ models predict the onset of star formation at $z \sim 25-31$, depending on the adopted cosmology. In these models, stars are formed automatically when the gas density increases above the adopted threshold, regardless of the time between the moment when the threshold is reached and the effective runaway collapse. While this is a reasonable approximation at low redshift, at high redshift this time interval represents a significant fraction of the Hubble time and thus this assumption can induce large artificial offsets to the onset of star formation. Choosing higher density thresholds $\left(135 \mathrm{~h}^{2} \mathrm{~cm}^{-3}\right)$ allows the entire cooling process to be followed, and the onset of star formation is then estimated to be at redshift $z \sim 12-16$. When isolated, rare, high-density peaks are considered, the chemical evolution is much faster and the first star formation episodes occur at $z \gtrsim 40$, almost regardless of the choice of the density threshold.

Conclusions. These results could have implications for the formation redshift of the first cosmological objects, as inferred from direct numerical simulations of mean-density environments and studies of the reionization history of the universe.
\end{abstract}

Key words. methods: $N$-body simulations - large-scale structure of Universe - early Universe

\section{Introduction}

Understanding primordial structure formation is one of the fundamental issues of modern astrophysics and cosmology. There is wide agreement that not only consists the universe of ordinary "baryonic" matter but also a large fraction of unknown "dark" matter, whose effects are only gravitational. Baryonic matter appears to constitute only a small fraction of the total cosmological matter content with a present-day density parameter $\Omega_{0, \mathrm{~b}}=0.0441$ compared to $\Omega_{0, \mathrm{~m}}=0.258$ (Hinshaw et al. 2008). Since the universe is observed to have zero curvature, i.e. to have a total density parameter $\Omega_{0, \text { tot }}=1$, these data imply that an additional density term exists $\Omega_{0, \Lambda}=0.742$. This is probably related to the so-called "cosmological constant" (Einstein 1917), or, as initially suggested by Ratra \& Peebles (1988), Wetterich (1988), Brax \& Martin (1999) and Peebles \& Ratra (2003), to other kinds of unknown "dark energies", whose effects on early structure formation history have been studied by e.g. Maio et al. (2006) with numerical simulations and by Crociani et al. (2008) with analytical calculations.

The existence of non-baryonic matter was suggested several decades ago, and structure formation models based on the growth of primordial gravitational instabilities (Peebles 1974; White \& Rees 1978) were developed following the early work by Gunn \& Gott (1972).

Hydrodynamical simulation codes (the first dating back to Evrard 1988; and Hernquist \& Katz 1989) have become a powerful tool, but because of computational limitations, plausible subgrid models have always been required to take into account star formation events (e.g. Cen \& Ostriker 1992; Katz 1992; Katz et al. 1996; Springel \& Hernquist 2003; Dalla Vecchia \& Schaye 2008; Schaye \& Dalla Vecchia 2008). These simulations model the converging gas infall into dark-matter potential wells, by following the gas that becomes shock heated and subsequently cools by atomic and/or molecular cooling. Given the many orders of magnitude (in scale and density) spanned, it is computationally extremely challenging to simulate the process down to the formation of single stars.

The gas physics in structure formation simulations has been typically approached with either lagrangian smoothed particle hydro-dynamics (SPH) or Eulerian mesh codes. A particular subclass is constituted by adaptive mesh refinement (AMR) codes, which allow further decomposition of the mesh around high-density regions, achieving a higher resolution. The main advantage of the SPH approach is its ability to follow self gravity in detail, while hydrodynamical instabilities are usually captured by mesh codes.

To account for star-formation episodes, both SPH and mesh schemes rely on specific assumptions. The prescriptions applied in mesh codes (e.g. Cen \& Ostriker 1992; Inutsuka \& Miyama 1992; Truelove et al. 1997) usually assume that the star formation rate is proportional to the density of overdense gas, while those used in SPH codes (e.g. Katz 1992; Bate \& Burkert 1997; 
Springel \& Hernquist 2003) are based on the existence of a density threshold above which the gas is gradually converted into stars. Here we make use of SPH simulations.

The typical timescales involved in the process of gas condensation are the free-fall time, $t_{\mathrm{ff}}$, and the cooling time, $t_{\text {cool }}$. Gas condensation is expected to take place only if $t_{\mathrm{cool}}<t_{\mathrm{ff}}$.

The free-fall time is defined as

$t_{\mathrm{ff}}=\sqrt{\frac{3 \pi}{32 G \rho}}$,

where $G$ is the universal gravitational constant and $\rho$ the density of the medium; the numeric factor $(3 \pi / 32)^{1 / 2}$ is exact for spherical symmetry only. The cooling time is defined as

$t_{\text {cool }}=\frac{3}{2} \frac{n k_{\mathrm{B}} T}{\mathcal{L}\left(T, n_{i}\right)}$,

where $n$ is the number density of the gas, $k_{\mathrm{B}}$ the Boltzmann constant, $T$ the temperature, and $\mathcal{L}\left(T, n_{i}\right)$ the cooling function (energy emitted per unit time and volume), which is dependent on both temperature and number densities, $n_{i}$, of the species constituting the gas. In the low-density limit ${ }^{1}$, for two-body interactions, between particles $x$ and $y, \mathcal{L}$ can be written as

$$
\mathcal{L}\left(T, n_{x}, n_{y}\right)=\Lambda(T) n_{x} n_{y}
$$

with the quantum-mechanical function $\Lambda(T)$ depending on the temperature of the species considered, $n_{x}$ and $n_{y}$ (see for example Maio et al. 2007). At $T \geq 10^{4} \mathrm{~K}$, the cooling is dominated by collisions of hydrogen atoms, which is the most abundant species in nature - about $93 \%$ in number fraction - and $\mathcal{L}$ scales approximatively as $n_{\mathrm{H}}^{2}$ (we indicate with $n_{\mathrm{H}}$ the hydrogen number density).

The physical conditions in which the first structures form are characterized by a primordial chemical composition: mostly hydrogen, deuterium, helium, and some simple molecules, e.g. $\mathrm{H}_{2}$ and $\mathrm{HD}$.

The primordial sites in which the first stars form are thought to be small dark-matter haloes with masses $\sim 10^{6} M_{\odot}-$ as expected from predictions based on self-similar gravitational condensation and chemical evolution (e.g. Tegmark et al. 1997; Trenti \& Stiavelli 2009) - and virial temperatures $T_{\text {vir }} \lesssim 10^{4} \mathrm{~K}$. Once they are born, they illuminate the universe and mark the end of the "dark ages". The radiation propagates in the vicinity of the individual sources and the impact on the subsequent structure formation (Ricotti et al. 2002a,b, 2008) can be very significant leaving imprints by a means of feedback effects (see Ciardi \& Ferrara 2005, for a review).

The low virialization temperatures of primordial haloes are enough neither to excite nor to ionize hydrogen and the lack of any metals means that the gas can cool and eventually form objects only via molecular transitions (Saslaw \& Zipoy 1967; Peebles \& Dicke 1968; Hollenbach \& McKee 1979, Maio et al. 2007, for a detailed study of the cooling efficiency in different regimes). They have rotational energy separations with excitation temperatures below $10^{4} \mathrm{~K}$, and therefore it is possible to collisionally populate their higher levels with the consequent emission of radiation and resulting gas cooling. Since the

\footnotetext{
1 This widely-used approximation is appropriate as, according to the classical spherical "top-hat" model, a virialized object has a total mass density of $18 \pi^{2}$ times the critical density, which corresponds, on average, to a total number density of $\sim 2 h^{2} \mathrm{~cm}^{-3}$ at $z \sim 15$, for a WMAP5 cosmology and a mean molecular weight $\mu \simeq 1$. The transition to a high-density statistical equilibrium regime happens at critical number densities of $\sim 10^{4} \mathrm{~cm}^{-3}$.
}

molecular energy-state separations are typically smaller than the atomic ones, cooling will of course be slower, but still capable of bringing the temperature down to $\$ 10^{2} \mathrm{~K}$ (Yoshida et al. 2003; Omukai \& Palla 2003; Yoshida et al. 2006; Gao et al. 2007).

To follow the entire process of structure and star formation in numerical simulations, one should implement the entire set of chemical reactions and hydrodynamical equations and from those calculate the abundance evolution and the corresponding cooling terms. In practice, performing these computations is very expensive and time consuming and it becomes extremely challenging to follow the formation of structures from the initial gas infall into the dark-matter potential wells to the final birth of stars. Nevertheless, efforts are being made in this direction (e.g. Abel et al. 2002; Bromm \& Larson 2004; Yoshida et al. 2007; Whalen et al. 2008).

For this reason, more practical, even if sometimes coarse, simple models are adopted. In brief, star formation relies on semi-empirical and numerical recipes based on chosen criteria to convert gas into stars and obtain the star formation rate, carefully normalized to fit observational data at the present day. In particular, in SPH approaches a single particle represents a population of stars with assigned mass distribution. The standard method used is to assume that once the gas has reached a given density threshold it automatically forms stars ${ }^{2}$ (e.g Cen \& Ostriker 1992; Katz 1992; Katz et al. 1996, and the popular Springel \& Hernquist 2003, model, inspired by the previous works), regardless of the time between the moment when the threshold is reached and the effective run-away collapse, which typically takes place at densities $\sim 10^{2}-10^{4} \mathrm{~cm}^{-3}$. While this is a reasonable approximation at low redshift, in "average" regions of the universe at high redshift this time interval represents a significant fraction of the Hubble time and thus the assumption can induce large artificial offsets on the onset of star formation and influence the evolution in the derived star formation rate. Thus, extrapolations to high redshifts of the low-density thresholds (few $10^{-2} \mathrm{~cm}^{-3}$ ) used to model the star formation rate in the low-redshift universe, may not always be justifiable. For this reason, high-redshift applications require higher resolutions and a higher density threshold.

In this paper, we are interested in modeling star formation as a global process in regions of mean density in the universe, not directly in the very first stars, which instead form in highly overdense, isolated regions. In particular, we discuss the importance of the choice of the density threshold for star formation in simulations of early structure formation. We present a criterion to choose this threshold (Sect. 2) and some test cases based on high-resolution simulations (Sects. 3 and 4). Then we present our results and conclusions (Sect. 5).

\section{Threshold for star formation}

According to the usual scenario of structure formation, the Jeans mass (Jeans 1902) is the fundamental quantity that allows us to distinguish collapsing from non-collapsing objects, under gravitational instability. For a perfect, isothermal gas, it is given by

$M_{\mathrm{J}}=\frac{\pi}{6}\left(\frac{k_{\mathrm{B}} T}{\mu m_{\mathrm{H}} G}\right)^{3 / 2} \rho^{-1 / 2}$,

where $m_{\mathrm{H}}$ is the mass of the hydrogen atom, and $T$ and $\rho$ are the temperature and density of the gas, respectively. At

\footnotetext{
2 To reduce the computation time for calculations of fragmentation sometimes, particles with densities above the threshold are replaced by "sink" particles (Bate et al. 1995).
} 
very high redshift $(z \sim 30-20)$, typical haloes have masses of $\sim 10^{5}-10^{6} M_{\odot}$, which can increase up to $\sim 10^{8}-10^{9} M_{\odot}$ by $z \sim 10$.

As mentioned in the introduction, the density threshold for star formation in numerical simulations is typically fixed to some constant value, irrespective of the simulation resolution. However, it would be desirable to have a star formation criterion that allows us to reach scales that fully resolve the Jeans mass.

The SPH algorithm implicitly imposes a minimum mass resolution limit, because to compute the different physical quantities, a fixed number of neighbours (i.e. number of particles within the smoothing length $\left.{ }^{3}, h\right)$ is used. This also induces a minimum resolvable mass, which is the total mass of neighbouring particles. This is particularly important in SPH simulations of cosmological structures and galaxy formation, because only if the minimum resolvable mass is far smaller than the Jeans mass, it is possible to ensure that the results are not affected by numerics nor by the details of the implementation adopted (Bate \& Burkert 1997). Otherwise, unresolved, Jeans unstable clumps can easily be found to exhibit unphysical behaviour (e.g. over-fragmentation problem in low-resolution simulations). Furthermore, it was shown (Navarro \& White 1993; Bate \& Burkert 1997) that the minimum number of particles needed to obtain reasonable and converging results is about twice the number of neighbours $\left(\sim 10^{2}\right.$ particles $)$.

If $M_{\text {res }}$ is the gas mass resolution of a given simulation, we can assume that:

$M_{\mathrm{J}}=N M_{\mathrm{res}}$,

where $N \gg 1$ and that the critical threshold is

$$
\begin{aligned}
\rho_{\text {th }} & =\frac{\pi^{2}}{36 N^{2} M_{\mathrm{res}}^{2}}\left(\frac{k_{\mathrm{B}} T}{\mu m_{\mathrm{H}} G}\right)^{3} \\
& \simeq \frac{1.31 \times 10^{-13}}{N^{2}}\left(\frac{M_{\mathrm{res}}}{M_{\odot}}\right)^{-2}\left(\frac{T}{10^{3} \mathrm{~K}}\right)^{3}\left(\frac{1}{\mu}\right)^{3}\left[\mathrm{~g} \mathrm{~cm}^{-3}\right] .
\end{aligned}
$$

For $M_{\text {res }}=10^{2} M_{\odot}, T=10^{3} \mathrm{~K}$, and $\mu=1$ and when using $N=10^{2}$ gas particles, one has $\rho_{\mathrm{th}} \sim 10^{-21} \mathrm{~g} \mathrm{~cm}^{-3}$, corresponding to a physical number density of $\sim 10^{2} \mathrm{~cm}^{-3}$. The above equations should be considered as a guideline to estimating the density threshold. We note that we largely fulfill the Bate \& Burkert (1997) conditions, because the neighbour number in our simulations is $N_{\text {neigh }}=32$ (see Sect. 3), so we resolve the Jeans mass with about three times the number of neighbours $(N \simeq$ $\left.3 N_{\text {neigh }}\right)$. As already mentioned however, commonly adopted density thresholds are for practical reasons usually chosen to be of order of $\sim 10^{-1} \mathrm{~cm}^{-3}$ or less (Katz et al. 1996; Springel \& Hernquist 2003; Scannapieco et al. 2005; Governato et al. 2007; Tornatore et al. 2007b; Schaye \& Dalla Vecchia 2008). For example, Wiersma et al. (2009) used a number density threshold of $10^{-1} \mathrm{~cm}^{-3}$, while the gas-particle mass was close to be the Jeans mass and the gravitational softening of order of the Jeans length.

In general, in simulations of both cosmic structure and galaxy formation, it is quite hard to fully resolve the Jeans mass of the collapsing fragments, and star formation is often assumed to occur while the gas falling into the dark-matter potential wells is still heating up. The Bate \& Burkert (1997) requirement is not usually satisfied, since the main goal is usually not to follow the entire process of collapse and fragmentation, but to obtain

\footnotetext{
3 The definition of smoothing length may vary from authors to authors. It is sometimes meant to be the width of the SPH smoothing kernel, but other times the length scale on which the SPH smoothing kernel becomes zero.
}

a qualitatively representative sample of the cosmological evolution.

A high value for the threshold is also important to capture the relevant phases of cooling. In the following, we show that molecule radiative losses, at temperatures of $\sim 10^{3}-10^{4} \mathrm{~K}$ where they can balance the heating of the infalling gas, produce an isothermal state and a subsequent cooling regime. For a region of mean density, the time spent by the gas in the isothermal state can be a substantial fraction of the Hubble time. Therefore, it is important for the threshold to be on the right-hand side of the peak in the phase-diagram (i.e. at densities higher than the isothermal regime), so that the delay between reaching the threshold and the true star formation is negligible (see Sect. 4).

In the following, we investigate the effect of different choices of star formation thresholds at high redshift, describe the simulations performed, and discuss the results obtained.

\section{Simulation set-up}

To study the effect of different threshold prescriptions on the onset of star formation, we completed very high resolution, three-dimensional, hydrodynamic simulations including nonequilibrium atomic and molecular chemistry, star formation, and wind feedback.

We used the code Gadget-2 (Springel 2005) in its modified form, which includes stellar evolution and metal pollution (Tornatore et al. 2007a), primordial molecular chemistry (following the evolution of $\mathrm{e}^{-}, \mathrm{H}, \mathrm{H}^{+}, \mathrm{He}, \mathrm{He}^{+}, \mathrm{He}^{++}, \mathrm{H}_{2}, \mathrm{H}_{2}^{+}, \mathrm{H}^{-}$, $\mathrm{D}, \mathrm{D}^{+}, \mathrm{HD}, \mathrm{HeH}^{+}$), and fine structure metal transition cooling $\left(\mathrm{O}, \mathrm{C}^{+}, \mathrm{Si}^{+}, \mathrm{Fe}^{+}\right)$at temperatures lower than $10^{4} \mathrm{~K}$ (Maio et al. 2007, 2008). We perform hydro-calculations by fixing the number of SPH neighbours to $N_{\text {neigh }}=32$.

The simulations have a comoving box size of $L=1 \mathrm{Mpc}$ and sample the cosmological medium with a uniform realization of $320^{3}$ particles for both gas and dark-matter species (for a total number of $2 \times 320^{3}$ particles). The resulting gas-particle mass is of the order of $10^{2} M_{\odot}$, which is consistent with the discussion in the previous section.

We note that this configuration enables us to easily resolve the Jeans length for shock heated/cooling cosmic gas (the Jeans length for gas with $T \sim 10^{4} \mathrm{~K}$ and $\rho \sim 10^{-25}-10^{-24} \mathrm{~g} / \mathrm{cm}^{3}$ is $\sim 3-1 \mathrm{kpc}$, much longer than the comoving gravitational softening $^{4}$ of $\left.\sim 0.1 \mathrm{kpc}\right)$.

The initial conditions (set at redshift $z=100$ ) are generated with a fast Fourier transform grid of $N_{\text {mesh }}=320$ meshes and a maximum wave-number (Nyquist frequency)

$k_{\text {Nyquist }}=\frac{2 \pi N_{\text {mesh }}}{2 L} \simeq 1 \mathrm{kpc}^{-1}$

(i.e. a minimum wavelength of $2 L / N_{\text {mesh }} \simeq 6.25 \mathrm{kpc}$ ), so that, for each wave-number, $\|\vec{k}\|<k_{\text {Nyquist }}$.

We will refer to this sampling as "mean region".

We considered two different sets of cosmological parameters:

- standard model: $\Omega_{0, \mathrm{~m}}=0.3, \Omega_{0, \Lambda}=0.7, \Omega_{0, \mathrm{~b}}=0.04, h=$ $0.7, \sigma_{8}=0.9$ and $n=1$, where the symbols have the usual meanings. The corresponding dark-matter and gas-particle masses are $\sim 755 M_{\odot} / h$ and $\sim 116 M_{\odot} / h$, respectively.

4 The comoving gravitational softening is usually estimated as $1 / 20$ or $1 / 30$ the mean inter-particle separation. 
Table 1. Parameters adopted for the simulations.

\begin{tabular}{lcccccccccr}
\hline \hline Model & $\begin{array}{c}\text { Number of } \\
\text { gas+dm particles }\end{array}$ & $\begin{array}{c}M_{\text {gas }} \\
{\left[M_{\odot} / h\right]}\end{array}$ & $\begin{array}{c}M_{\mathrm{dm}} \\
{\left[M_{\odot} / h\right]}\end{array}$ & $\Omega_{0 \mathrm{M}}$ & $\Omega_{0 \Lambda}$ & $\Omega_{0 \mathrm{~b}}$ & $h$ & $\sigma_{8}$ & $n$ & $\begin{array}{r}\text { SF threshold } \\
{\left[h^{2} \mathrm{~cm}^{-3}\right]}\end{array}$ \\
\hline wmap5-ht & $2 \times 32768000$ & 128 & 621 & 0.258 & 0.742 & 0.0441 & 0.72 & 0.8 & 0.96 & 135.0 \\
wmap5-lt & $2 \times 32768000$ & 128 & 621 & 0.258 & 0.742 & 0.0441 & 0.72 & 0.8 & 0.96 & 0.2 \\
std-ht & $2 \times 32768000$ & 116 & 755 & 0.300 & 0.700 & 0.0400 & 0.70 & 0.9 & 1.00 & 135.0 \\
std-lt & $2 \times 32768000$ & 116 & 755 & 0.300 & 0.700 & 0.0400 & 0.70 & 0.9 & 1.00 & 0.2 \\
zoom-std-ht & $2 \times 41226712$ & 3.9 & 25.6 & 0.300 & 0.700 & 0.0400 & 0.70 & 0.9 & 1.00 & 135.0 \\
\hline
\end{tabular}

The columns (from left to right) specify: name of the run, number of particles used, gas-particle mass, dark-matter-particle mass, $\Omega_{0, \mathrm{~m}}, \Omega_{0, \Lambda}, \Omega_{0, \mathrm{~b}}$, $h, \sigma_{8}$, spectral index, star formation density threshold.

- WMAP5 model: data from 5-year WMAP (WMAP5) satellite (Hinshaw et al. 2008) suggest that $\Omega_{0, \mathrm{~m}}=0.258, \Omega_{0, \Lambda}=$ $0.742, \Omega_{0, \mathrm{~b}}=0.0441, h=0.72, \sigma_{8}=0.796$, and $n=0.96$. In this case, the corresponding dark-matter and gas-particle masses are $\sim 621 M_{\odot} / h$ and $\sim 128 M_{\odot} / h$, respectively.

Following the discussion in the previous sections, we also consider two different values for the star formation density threshold:

- a low-density threshold of $0.2 h^{2} \mathrm{~cm}^{-3}$ (physical), compatible with the one adopted in the Gadget code and the ones widely used in the literature (for example Katz et al. 1996; Springel \& Hernquist 2003; Tornatore et al. 2007b; Pawlik et al. 2009);

- a high-density threshold of $135 \mathrm{~h}^{2} \mathrm{~cm}^{-3}$ (physical), as computed from Eqs. (6) and (7). This value is adequate for modelling atomic processes even in small $\sim 10^{5} M_{\odot}$ haloes at $z \sim 20$. Moreover, this threshold typically falls in density regimes where cooling dominates over heating, allowing us to properly resolve gas condensation down to the bottom of the cooling branch.

A summary of all the simulation features is given in Table 1. We denote with the labels "std" and "wmap5" the runs with standard and WMAP5 cosmology, respectively, and with "lt" and "ht" the runs with low- and high-density thresholds, respectively.

We note that the Springel \& Hernquist (2003) model used here to describe the star formation process is strictly applicable only as long as more than one star per SPH particle is present, i.e. each SPH particle is considered as a "simple stellar population $\tilde{A}$ " with a given mass distribution. Although some studies (Yoshida et al. 2003; Bromm et al. 2002; Bromm \& Larson 2004) seem to indicate (or assume) that the very first episode of star formation could result in a single, very massive star per halo, this should apply preferentially to very high redshift, high density, isolated objects. In any case, the exact shape of the IMF of primordial stars (e.g. Schwarzschild \& Spitzer 1953; Larson 1998; Nakamura \& Umemura 2001; Omukai \& Palla 2003) is still a matter of speculation and lively debate. For this reason and because we are interested mainly in the global star formation process, we used the Springel \& Hernquist (2003) model (as Tornatore et al. 2007b, also did to describe both a primordial topheavy and a more standard star formation mode) and allowed the IMF to be a free parameter (although in the test cases reported here we always adopt a top-heavy IMF). The aim of this paper is to investigate the effects of the density threshold on star formation, and we leave discussion on the IMF to future work.

Finally, to investigate primordial star formation events in local high-density regions, we perform a very high-resolution numerical simulation of a rare high-sigma peak with comoving radius $\sim 140 \mathrm{kpc} / h$. This region is selected using the zoomed initial condition technique on a $\sim 10^{9} M_{\odot}$ halo formed in a darkmatter-only simulation (Gao et al. 2007) ${ }^{5}$. We divided each particle into gas and dark-matter component, according to the standard model parameters. The resulting gas-particle mass is $\sim 4 M_{\odot} / h$ and dark matter particles have a mass of $\sim 26 M_{\odot} / h$ (in Table 1, this simulation is labelled "zoom-std-ht").

By a quick comparison of the different parameters adopted, we expect that, once the density threshold has been fixed, the standard cosmological mean-region simulations will show earlier structure formation episodes with respect to the corresponding wmap5 ones. This is because they have higher spectral parameters and higher matter content. The high-density region is a biased over-dense region already at early times, and therefore, its evolution is expected to be much faster.

\section{Results}

We present the results of the simulations with the sets of parameters described above. We discuss first the mean region of the universe (Sect. 4.1) and then the high-density region (Sect. 4.2).

\subsection{Mean-region simulation}

Our reference run is the wmap5-ht model with initial composition given by the values quoted in Galli \& Palla (1998) ${ }^{6}$ at $z=100$. We show some evolutionary stages in Fig. 1 (upper set of panels). In the maps, the first column refers to temperature, the second to gas density and the third to molecular fraction at $z=30.16$ and $z=12.17$, respectively. The creation of new molecules is clearly evident, together with the related growth of structures. More specifically, as time passes, one can see the heating undergone by the gas in dense regions, because of structure formation shocks. The temperature increases from a few hundreds Kelvin in the low-density regions, to $\sim 10^{4} \mathrm{~K}$ in the denser regions. In the meantime, the molecular fraction also evolves accordingly up to values higher than $10^{-4}$. Soon after, the production of molecules increases rapidly (up to $\sim 10^{-2}$ ) aiding the star formation process, which, for this simulation, starts at $z \simeq 12$.

In Fig. 2, we show the phase diagram (comoving density versus temperature) at redshift $z \simeq 12$, i.e. just before the onset of star formation. The low-density gas, which is shock-heated by the collapse of the first primordial haloes, is seen on the left side of the panel. Starting from values for a temperature of $\sim 10^{2} \mathrm{~K}$,

\footnotetext{
5 We use the "R4" initial conditions presented there.

6 We assume a primordial neutral gas with residual electron and $\mathrm{H}^{+}$ fractions $x_{\mathrm{e}^{-}} \simeq x_{\mathrm{H}^{+}} \simeq 4 \times 10^{-4}, \mathrm{H}_{2}$ fraction $x_{\mathrm{H}_{2}}=10^{-6}, \mathrm{H}_{2}^{+}$fraction $x_{\mathrm{H}_{2}^{+}}=3 \times 10^{-21}$, D fraction $x_{\mathrm{D}}=3.5 \times 10^{-5}$, HD fraction $x_{\mathrm{HD}}=$ $7 \times 10^{-10}, \mathrm{D}^{+}$fraction $x_{\mathrm{D}^{+}}=4 \times 10^{-9}, \mathrm{HeH}^{+}$fraction $x_{\mathrm{HeH}^{+}}=10^{-14}$.
} 
$\log _{10}(\mathrm{~T} / \mathrm{K})$
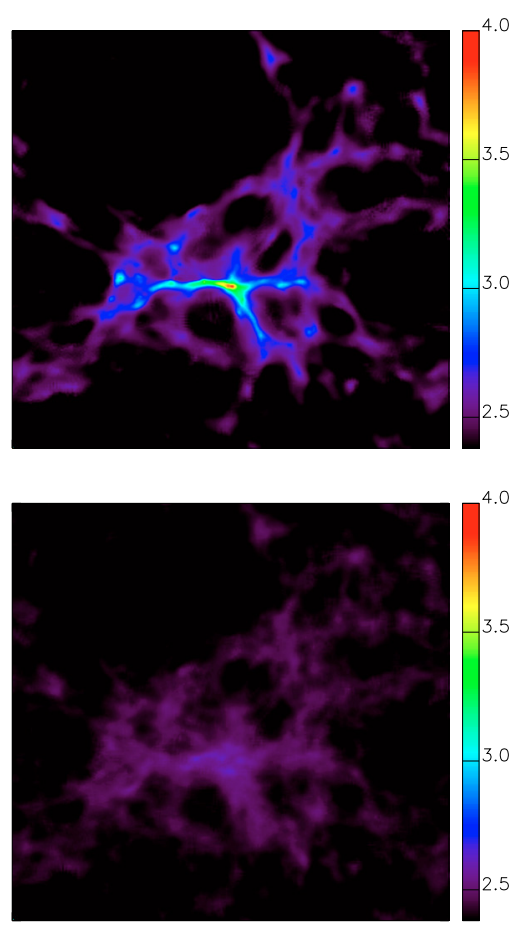

$\log _{10}(\mathrm{~T} / \mathrm{K})$
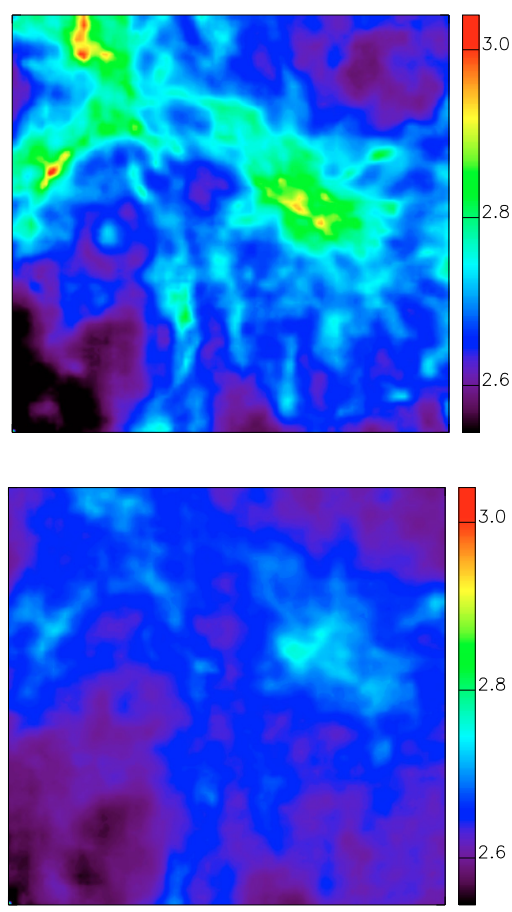

\section{Mean-region maps}

$\log _{10}\left(\rho_{\text {com }} / h^{2} \mathrm{~g} \mathrm{~cm}^{-3}\right)$
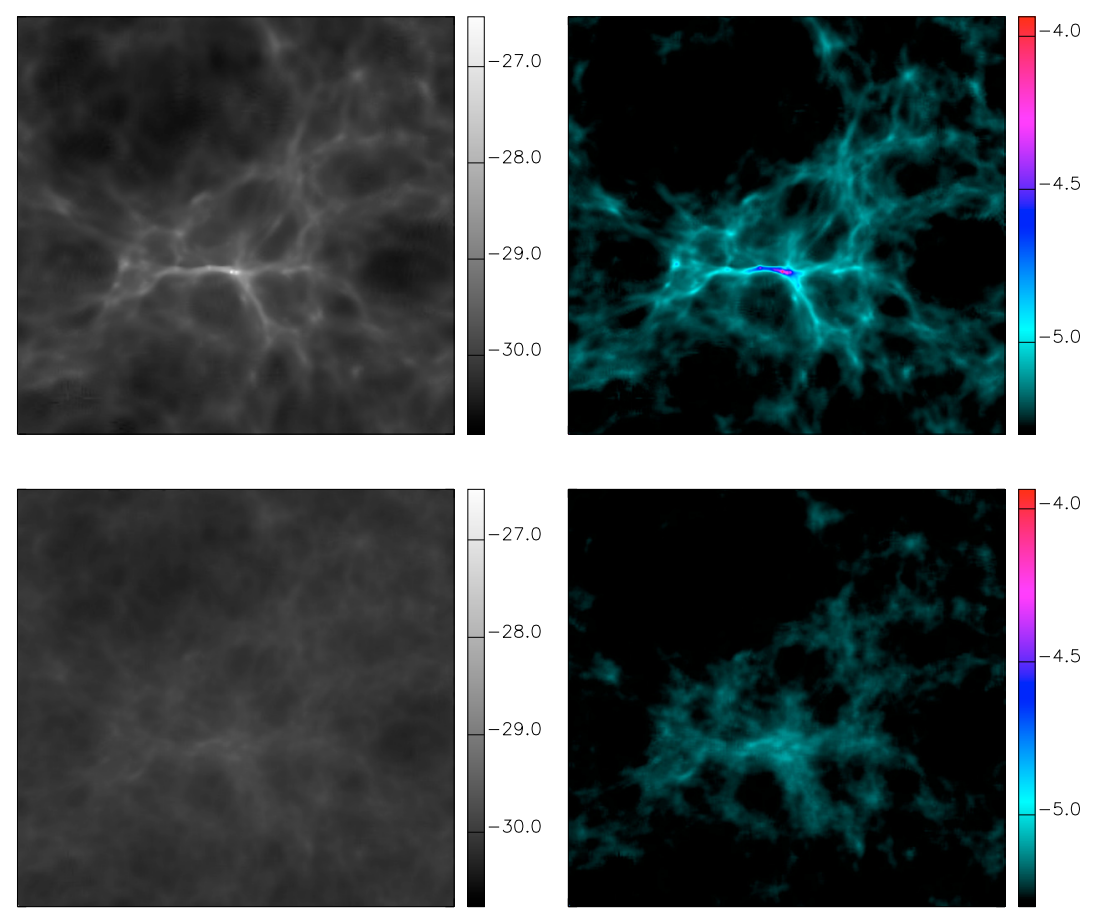

High-density region maps $\log _{10}\left(\rho_{\text {com }} / h^{2} \mathrm{~g} \mathrm{~cm}^{-3}\right)$
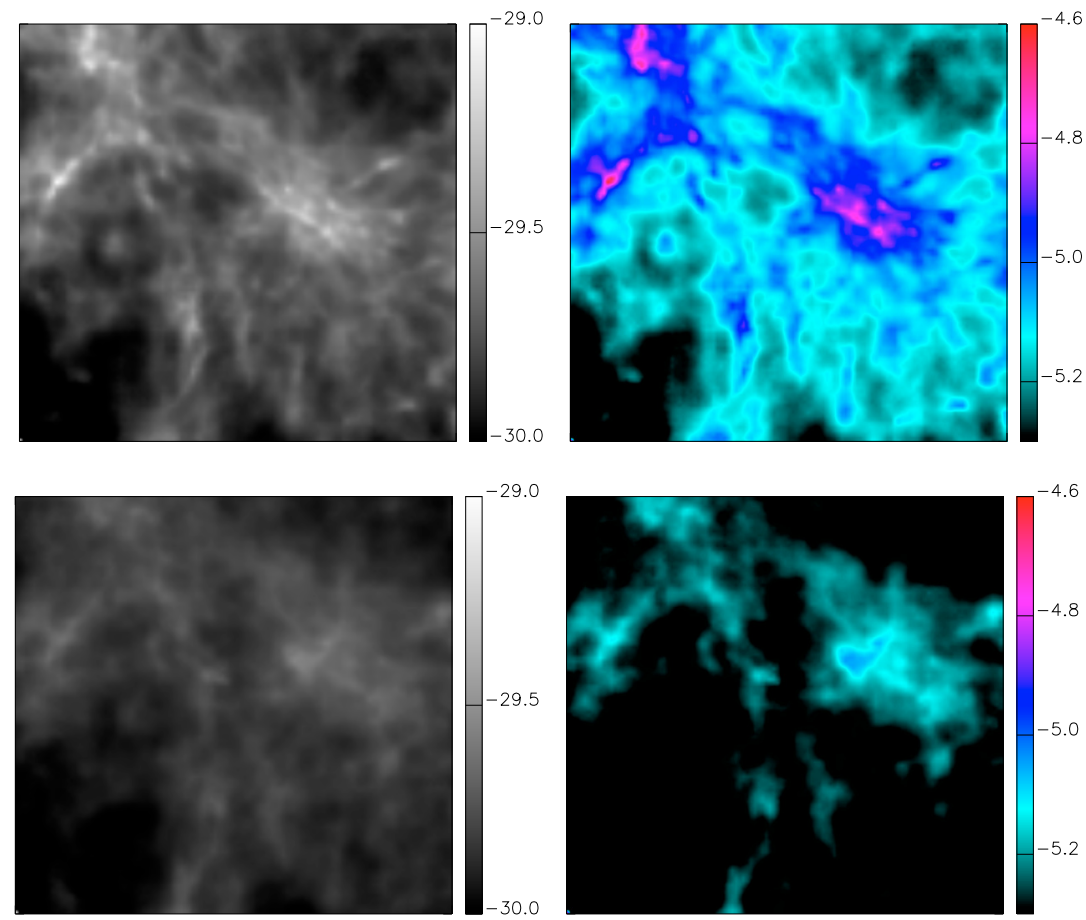

Fig. 1. First, second, and third column are respectively temperature, density, and molecule maps. The first two rows refer to the mean-region simulation at redshift 12.17 (top) and 30.16 (bottom). The box size is $1 \mathrm{Mpc}$ comoving. The last two rows refer to the high-density region at redshift 50 (top) and 70 (bottom). The region size is $\sim 140 \mathrm{kpc} / h$ comoving. All quantities are smoothed on a 276-pixel side grid. 


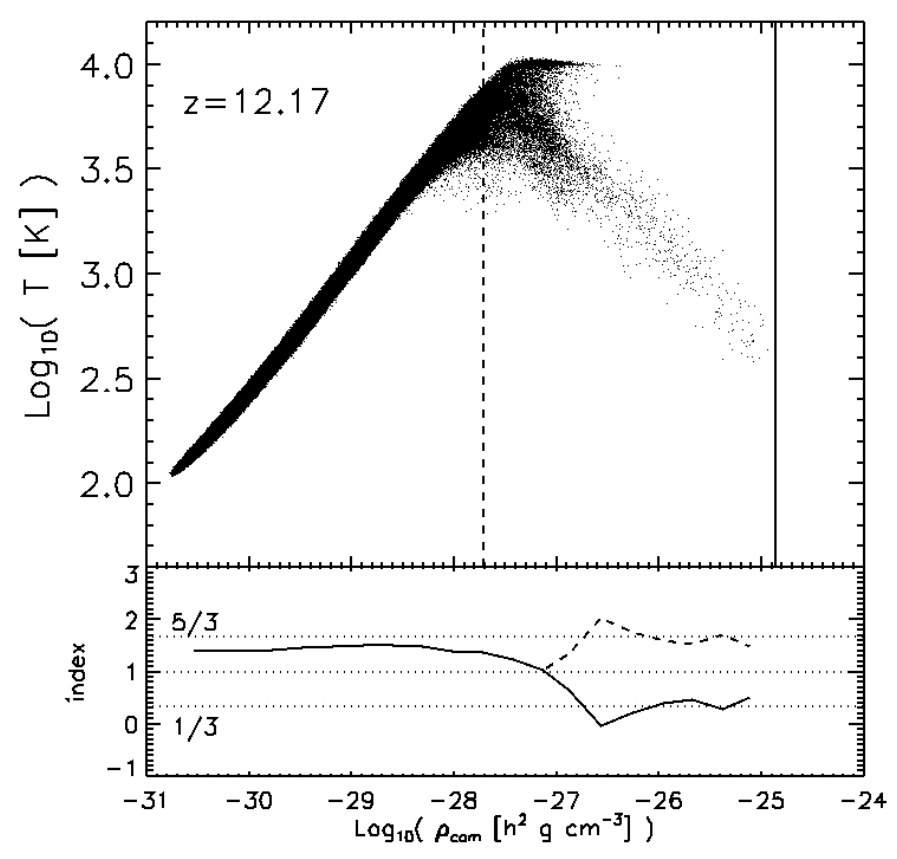

Fig. 2. Upper panel: phase diagram at redshift $z=12.17$ (just before the onset of star formation) for the wmap5-ht simulation. The vertical straight lines indicate a low physical critical density threshold of $0.2 \mathrm{~h}^{2} \mathrm{~cm}^{-3}$ (dashed line) and a higher physical critical density threshold of $135 h^{2} \mathrm{~cm}^{-3}$ (solid line). Lower panel: average effective index computed over the whole range of densities. The three horizontal dotted lines show values of $5 / 3,1$ and $1 / 3$, respectively from top to bottom. The solid line shows $\alpha$ and the dashed line shows $\gamma$ (see text for definitions).

the gas is progressively heated to $\sim 10^{4} \mathrm{~K}$ and moves along the rising branch. At this stage, collisions become more frequent due to the higher temperature. The upper energy levels of particles become excited and the subsequent de-excitation is accompanied by the emission of radiation. This effect is negligible at low densities, because collisions are rare and the fraction of energy converted into radiation is small. When the density increases, the cooling becomes comparable to the heating and an isothermal regime with no significant net change in the temperature is reached. This appears at the tip of the phase diagram (and in the behaviour of the effective index, as discussed below), at $T \sim 10^{4} \mathrm{~K}$, where the cooling is dominated by atomic Ly $\alpha$ transitions and accompanied by runaway collapse. At higher densities, radiative losses overtake heating and induce a fast cooling phase (dominated by molecules, mostly $\mathrm{H}_{2}$ ).

The solid vertical line corresponds to the physical highdensity star formation threshold $\left(135 \mathrm{~h}^{2} \mathrm{~cm}^{-3}\right)$ and, for comparison, we also plot the dashed line for a physical number density of $0.2 \mathrm{~h}^{2} \mathrm{~cm}^{-3}$. We stress that by adopting a low-density threshold for star formation one completely misses the isothermal and cooling part of the phase diagram, and thus a correct modeling of the cooling regions within the simulations. This can affect the onset of star formation, particularly at high redshift, when the time needed for the gas to evolve from the low-density threshold to the high-density threshold $\left(\sim 2 \times 10^{8} \mathrm{yr}\right)$ can be a substantial fraction of the Hubble time $\left(\sim 4 \times 10^{8} \mathrm{yr}\right.$ at $\left.z \sim 12\right)$. We note that the time elapsed between the attainment of the isothermal peak in the phase diagram and the end of the cooling branch is $\sim 6 \times 10^{7} \mathrm{yr}$. The evolution that follows the end of the cooling branch is characterized by the formation of a dense core, which accretes gas on free-fall timescales (Yoshida et al. 2006). This

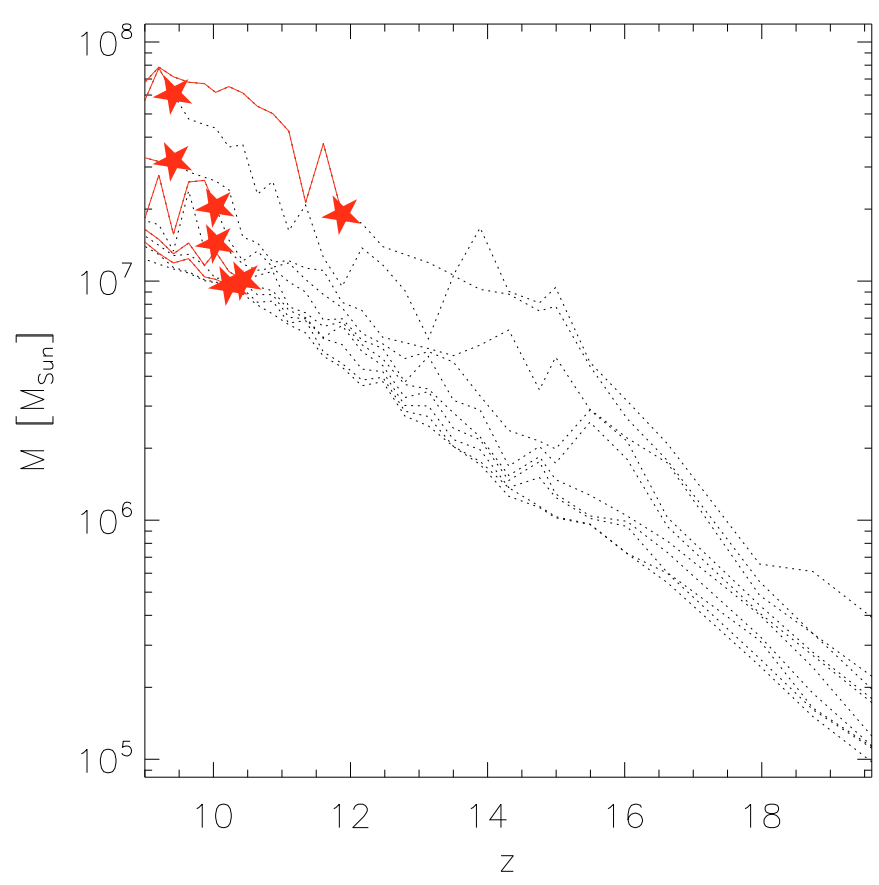

Fig. 3. Evolution of the ten most massive haloes in the wmap5-ht cosmological simulation (dotted lines). The redshift at which the first star forms in each halo is indicated by the filled star symbols. After that, star formation continues along the solid lines.

phase has a very short duration $\left(\sim 10^{6}-10^{7} \mathrm{yr}\right)$ during which the central densities increase to $\sim 10^{16} \mathrm{~cm}^{-3}$. The problem is less severe at lower redshift, when the Hubble time becomes of the order of several Gyr.

The density and temperature behaviour can also be described by an effective index ${ }^{7}$, which depends on the physical conditions of the gas regime considered. In the lower panel of Fig. 2, we plot the effective index as a function of density. The solid line refers to the value $\alpha \equiv 1+(\mathrm{d} T / T) /(\mathrm{d} \rho / \rho)$, which takes into account changes in the sign of the temperature derivative, distinguishing the heating regime $(\alpha>1)$ from the cooling regime $(\alpha<1)$. The dashed line refers to $\gamma \equiv 1+|(\mathrm{d} T / T) /(\mathrm{d} \rho / \rho)|$, so that $\gamma$ is always $\geq 1$. Dotted horizontal straight lines INDICATE values of $5 / 3,1$, and $1 / 3$. In correspondence with the isothermal peak in the $T-\rho$ plane, it is $\alpha=\gamma=1$, which marks the transition from the heating to the cooling regime. At this stage, we expect the gas runaway collapse to begin and last for the following cooling regime, at which point $\alpha$ oscillates around the value of $1 / 3$.

In Fig. 3, we plot the evolution of the ten most massive haloes found in the simulation. We also show the redshift at which stars are produced (filled star symbols) in each object. The haloes are found using a friend-of-friend algorithm with a linking length equal to $20 \%$ of the mean inter-particle separation. Typical halo masses at redshift $z \sim 12$, when star formation starts, are of the order of $10^{7} M_{\odot}$ (see also Wise \& Abel 2007, 2008) and reach densities of $\sim 10^{2} \mathrm{~cm}^{-3}$.

For comparison, we completed the same simulation using standard cosmological parameters (std-ht run). In this case, the overall picture is similar, but we detected a faster evolution, with earlier structure formation, as expected from the previous discussion in Sect. 3. The first star formation events are detected at redshift $z \sim 16$ in haloes with masses $\sim 10^{7} M_{\odot}$.

\footnotetext{
7 By effective index of the gas, $\gamma$, we mean $P \propto \rho^{\gamma}$, with $P$ pressure and $\rho$ density. This is simply related to the politropic index.
} 


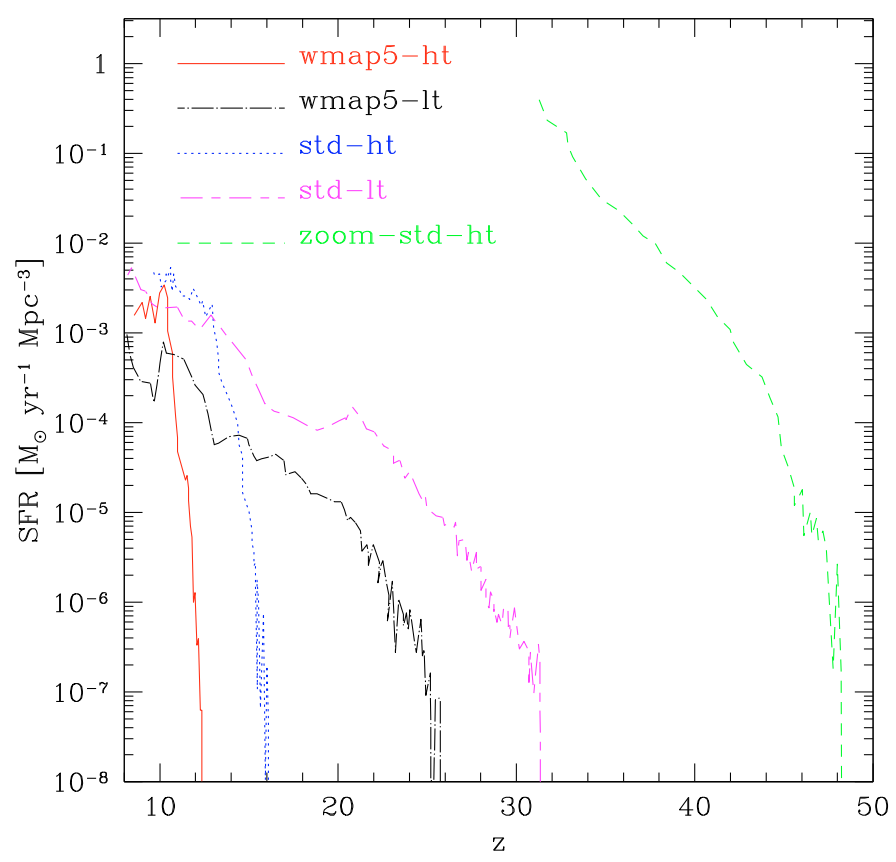

Fig. 4. Star formation rate as a function of redshift for the different models, from left to right: WMAP5 cosmology and high-density threshold (solid red line), standard cosmology and high-density threshold (dotted blue line), WMAP5 cosmology and low-density threshold (dot-dashed black line), standard cosmology and low-density threshold (long-dashed-short-dashed magenta line). The green short-dashed line refers to the simulation of the high-density region with standard parameters and high-density threshold.

This can clearly be seen in Fig. 4, where we plot the star formation rate as a function of redshift for the different simulations (to compute the star formation rate, we adopt the implementation described by Springel \& Hernquist 2003).

The onset of star formation in the wmap5-ht model (red solid line) is delayed compared to the std-ht model (blue dotted line). For the wmap5-1t (black dashed line) and std-lt (magenta shortlong-dashed line) models, star formation starts at $z \sim 25$ and 31, respectively. Thus, at these high redshifts, even small changes in the cosmology can be significant for the onset of star formation. This is easily understood in terms of spectral parameters: the standard cosmology has higher spectral index and normalization; therefore, assigning more power on all scales with respect to WMAP5 values, leads to structure formation occurring much earlier.

The choice of the density threshold makes an even larger difference to the onset of star formation. In Fig. 4, the rates corresponding to the wmap5-lt (black dot-dashed line) and wmap5-ht (red solid line) show that star formation starts at $z \sim 25$ and 12 , respectively. The major difference between low- and highdensity threshold models is that, in the former, the gas reaches the critical density much earlier. So, the redshift difference in the onset corresponds to the time that the gas needs to move from the low- to the high-density threshold (see Fig. 2).

In addition, the simulations adopting the high-density thresholds slightly overtake the respective low-threshold cases. This happens because the former did not remove the gas at higher redshifts, it accumulated and ended in delayed bursts of star formation. Later, the star formation rates were restored to the same level.

As already mentioned, the low-density threshold model is very commonly used both in numerical and semi-analytical works, because it does not require incorporation of molecular chemistry (the threshold being lower than the typical densities at which molecules become efficient coolants) and therefore it is easier to implement and allows faster simulations. However, it can compromise the entire picture if the results are extrapolated to high redshift, when molecules are the main coolants and the time delay between the attainment of the low-density threshold and the bottom of the cooling branch occupies a significant fraction of the Hubble time.

\subsection{High-density region simulation}

We show results for the high-density region described in Sect. 3 and initialized at redshift $z=399$.

In this case, the physical number densities at the beginning of the simulation are in the range $\sim 0.5-50 h^{2} \mathrm{~cm}^{-3}$ (at $z \sim 200$ ), with an average of $\sim 4 h^{2} \mathrm{~cm}^{-3}$, higher than the typical value adopted for the low-density threshold for star formation. Therefore, the conventional low-density model would produce unreasonable star formation at $z \sim 200$. To avoid this, it is common to add a further, additional, ad hoc constraint, which allows star formation only if the simulation over-densities are higher than a given minimum value - usually between $\sim 50$ and $\sim 100$ (Katz et al. 1996, in Sect. 4.2, for example, suggest 55.7). Thus, in this case it is this additional constraint that determines when the onset of star formation occurs, rather than the low-density threshold.

We therefore ran a simulation with only a high-density threshold. For the sake of comparison, we still used the value of $135 \mathrm{~h}^{2} \mathrm{~cm}^{-3}$, although rigorously, following Eqs. (6) and (7), one should adopt a value $\sim 9 \times 10^{4} h^{2} \mathrm{~cm}^{-3}$ for a $3.9 M_{\odot} / h$ gasparticle mass. Nonetheless, we checked that this choice does not affect our conclusions, since the threshold is already beyond the isothermal peak, in the fast cooling regime, where the timescales are extremely short $\left(\sim 10^{6} \mathrm{yr}\right)$. All the initial abundances are set according to the values suggested by Galli \& Palla $(1998)^{8}$.

The simulation maps are shown in Fig. 1 (lower panels). As for the mean-density regions, they refer to temperature, density, and molecular fraction at redshifts $z=50$ and 70. As expected, we highlight that structure formation occurs far earlier than the mean-density case. Molecular abundances of $\sim 10^{-5}$ are reached faster than for the mean-density region, where such a high fraction is found only at $z \lesssim 30$. Similarly, values of $\sim 10^{-4}$ are already reached at $z \sim 50-40$, rather than $z \sim 20-$ see also discussion in Sect. 5 and Eq. (9).

In Fig. 5, we show the phase diagram and the behaviour of the effective index as a function of the comoving gas density at redshift $z \simeq 45$. Physical critical density thresholds of $0.2 h^{2} \mathrm{~cm}^{-3}$ (dashed line), $135 h^{2} \mathrm{~cm}^{-3}$ (solid line), and $\sim 9 \times 10^{4} h^{2} \mathrm{~cm}^{-3}$ (dot-dashed line) are marked in the figure. While the first two are the same as for the mean-density region simulation, the last one corresponds to the value obtained using Eqs. (6) and (7). To emphasize the different characteristics of the phase diagram compared to the one obtained for the mean-density region, the plot was extended to densities higher than before. The isothermal peak is reached at redshift $z \sim 50$. Unlike the mean-density simulation, the gas does not spend time on the isothermal plateau, but cools very rapidly (in less than

\footnotetext{
8 The initial abundances (at $z=399$ ) are consistent with a primordial neutral plasma having residual electron and $\mathrm{H}^{+}$fractions of $x_{\mathrm{e}^{-}} \simeq x_{\mathrm{H}^{+}}=$ $10^{-3}, \mathrm{H}_{2}$ fraction $x_{\mathrm{H}_{2}}=10^{-10}, \mathrm{H}_{2}^{+}$fraction $x_{\mathrm{H}_{2}^{+}}=3 \times 10^{-15}$, D fraction $x_{\mathrm{D}}=3 \times 10^{-5}, \mathrm{D}^{+}$fraction $x_{\mathrm{D}^{+}}=3 \times 10^{-8}, \mathrm{HD}$ fraction $x_{\mathrm{HD}}=10^{-14}$, $\mathrm{HeH}^{+}$fraction $x_{\mathrm{HeH}^{+}}=5.6 \times 10^{-18}$.
} 


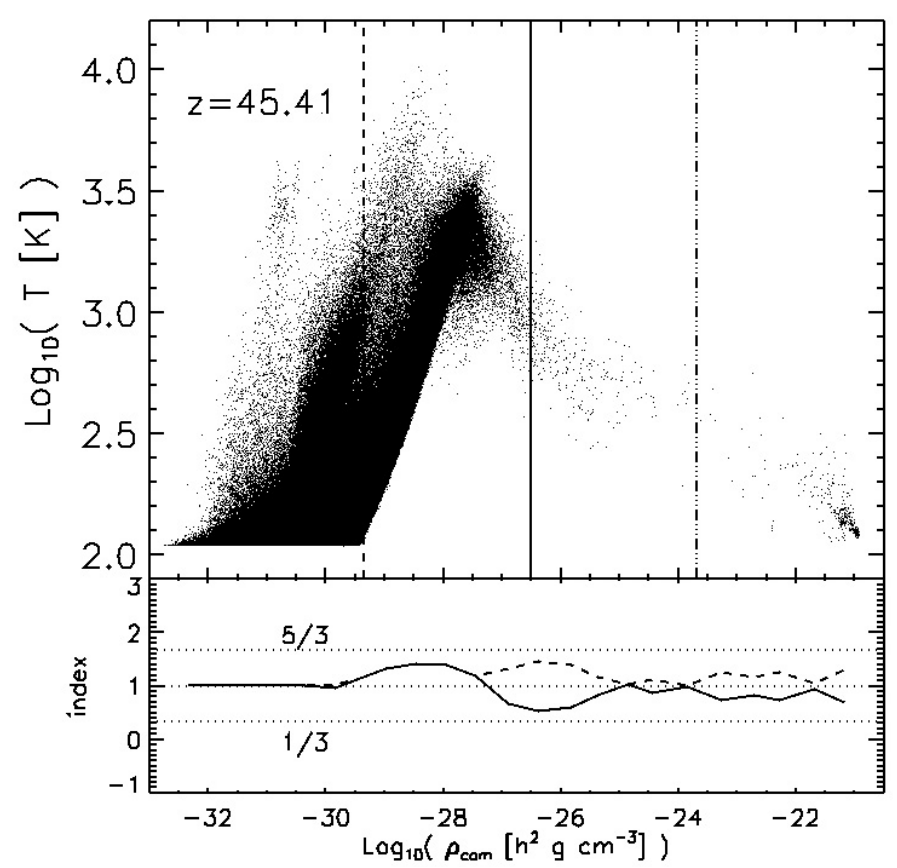

Fig. 5. Upper panel: phase diagram at redshift $z=45.41$ for the highdensity region simulation for a purely non-equilibrium chemistry run (i.e. without star formation). The vertical straight lines show physical critical-density threshold of $0.2 \mathrm{~h}^{2} \mathrm{~cm}^{-3}$ (dashed line), $135 \mathrm{~h}^{2} \mathrm{~cm}^{-3}$ (solid line) and $8.9 \times 10^{4} h^{2} \mathrm{~cm}^{-3}$ (dot-dashed line). Lower panel: average effective index computed over the whole range of densities. The three horizontal dotted lines show values of $5 / 3,1$, and $1 / 3$, respectively, from top to bottom. The solid line refers to $\alpha$ and the dashed line to $\gamma$ (see text for the definitions).

$7 \times 10^{6} \mathrm{yr}$ ) from $\sim 10^{3.5} \mathrm{~K}$ to $\sim 10^{2} \mathrm{~K}$ and condenses into comoving densities of $\rho_{\text {com }} \sim 10^{-21} h^{2} \mathrm{~g} / \mathrm{cm}^{3}$. The rapidity of these events is reflected in the lack of particles in the intermediate stages of the cooling branch.

As before, we also plot the effective gas index. The usual initial shock-heating behaviour and the following cooling is recovered up to much higher densities. At the bottom of the cooling branch, we find values of $\alpha$ that oscillate around $1 / 3$ and 1 . Since the last stages are quite fast, the low number of particles present introduces some statistical noise, which is evident in the plot.

With our choice of the threshold, star formation sets in at $z \sim$ 48 (see Fig. 4). The additional time needed to reach the highest densities at the bottom of the cooling branch is extremely short $\left(\sim 10^{6} \mathrm{yr}\right)$, so our choice ensures that the onset of star formation is correctly estimated. As there is no obvious, standard way of quantifying the star formation rate in these simulations, we do this by dividing the stellar mass formed at each time-step by the volume of the gas contained in the high-density region (a sphere of about $140 \mathrm{kpc} / h$ radius).

\section{Discussion and conclusions}

We have studied the effect of different choices of the density threshold on the onset of cosmic star formation in numerical SPH simulations (see Maio et al. 2007; Tornatore et al. 2007a, for technical details).

In the literature, several studies are presented that follow the birth of primordial stars in early protogalaxies (examples are Yoshida et al. 2006; Wise \& Abel 2007). These are mainly focused on the initial phases of star formation and the effects on the immediate surroundings, which typically do not address more general issues such as the global star formation process (i.e. in a region at mean density rather than in high density peaks), metal enrichment, and IGM reionization (e.g. Bolton \& Haehnelt 2007). Here instead, we are interested in simulating the more global star formation process in the high-redshift universe, capturing the relevant timescales and physical processes, i.e. the atomic and molecular physics that regulates the formation of primordial stellar population.

We have thus run simulations using initial conditions appropriate to a region of the universe with mean density and as a reference, using the zoom technique, a high-density peak.

A basic process that leads to star formation, i.e. gas shock heating up to $\sim 10^{3}-10^{4} \mathrm{~K}$ by infall into dark-matter haloes followed by radiative losses due mainly to molecular collisional excitations, is common to both scenarios. The main difference is associated with the global dynamics and timescales of the process. In the rare high-sigma peak, because of the higher densities, chemical reactions are faster and much more efficient with respect to the simulations of mean-density initial conditions. Therefore, the molecular fraction increases more rapidly, reaching a number fraction of $\sim 10^{-4}$ by $z \sim 50-40$ (compared to $z \sim 20$, for the corresponding mean-density case). These values are sufficient to make collisional cooling to dominate over heating and induce star formation episodes.

Density and temperature behaviour can be described by an effective index that depends on the physical conditions of the gas considered. Roughly, it is isothermal during the transition from the heating to the cooling regime, then it collapses (see the effective index computed in the lower panel of Fig. 2) until the bottom of the cooling branch is reached. More quantitatively, when cooling is dominated by $\mathrm{H}_{2}$, the cooling time in Eq. (2) can be approximated as

$t_{\text {cool }} \simeq \frac{3}{2} \frac{k_{\mathrm{B}} T}{\Lambda_{\mathrm{H}_{2}}(T) x_{\mathrm{H}_{2}} n_{\mathrm{H}}}$

where $x_{\mathrm{H}_{2}}$ is the $\mathrm{H}_{2}$ number fraction, $\Lambda_{\mathrm{H}_{2}}(T)$ is the $\mathrm{H}_{2}$ cooling function at temperature $T$, and the other symbols have their usual meanings.

For gas at the beginning of the cooling branch, $T \sim 10^{3.5} \mathrm{~K}$ and $x_{\mathrm{H}_{2}} \sim 10^{-4}$, giving $t_{\mathrm{cool}} \sim 7 \times 10^{6} n_{\mathrm{H}}^{-1} \mathrm{yr}\left(n_{\mathrm{H}}\right.$ in $\left.\mathrm{cm}^{-3}\right)$. In the mean-density case (see phase diagram in Fig. 2 ), $n_{\mathrm{H}} \simeq 0.3 \mathrm{~cm}^{-3}$, while in the high-density region (see phase diagram in Fig. 5), $n_{\mathrm{H}} \simeq 6 \mathrm{~cm}^{-3}$. This translates into a characteristic cooling time of $\sim 2 \times 10^{7} \mathrm{yr}$ for the former case and $\sim 10^{6} \mathrm{yr}$ for the latter.

These estimates show the relevance of following the full cooling branch when simulating star formation at high redshift in regions of mean density, because the characteristic cooling times are a substantial fraction of the Hubble time. This problem is less severe for simulations of high-density peaks, in which the timescales are much shorter.

If one considers a $10^{5} M_{\odot}$ halo at $z \sim 40$, its average gas number density is of the order of $\sim 20 / \mu \mathrm{cm}^{-3}$ (being $\mu$ the mean molecular weight), its virial temperature $T_{\text {vir }} \sim 7 \times 10^{2} \mu \mathrm{K}$ and its cooling time $t_{\text {cool }} \sim 3 \times 10^{5}-3 \times 10^{8}$ yr for an $\mathrm{H}_{2}$ fraction of $10^{-2}-10^{-5}$, respectively. The same halo at redshift $z \sim 15$ would have an average gas number density of $\sim 1 / \mu \mathrm{cm}^{-3}, T_{\text {vir }} \sim 5 \times$ $10^{2} \mu \mathrm{K}$, and $t_{\text {cool }} \sim 7 \times 10^{6}-7 \times 10^{9} \mathrm{yr}$. This means not only that objects of similar mass cool (and thus harbor star formation) on very different timescales according to their environment, but also that gas in high-redshift haloes can collapse and fragment much faster than in low-redshift ones, because its cooling capabilities are more efficient.

For these reasons, though low-density thresholds can be useful tools to reproduce empirical surface-density relations, 
such as the Kennicutt-Schmidt law (Kennicutt 1998; Springel \& Hernquist 2003; Schaye \& Dalla Vecchia 2008), physical insights can rely only on high-density thresholds. Indeed, imposing star formation events before the isothermal peak is reached could result in an artificially high-redshift for the onset of star formation.

For the test cases presented in this paper, the value adopted for the high-density threshold is $135 \mathrm{~h}^{2} \mathrm{~cm}^{-3}$, well beyond the isothermal peak of the gas. This allows a correct estimate of the relevant timescales, as the gas spends most of the time in the isothermal phase. In addition, following the evolution of the gas to higher densities allows higher resolution of, e.g., the morphology and disk galaxy structure (Saitoh et al. 2008), the clumpiness of the gas, and the features of the interstellar or intergalactic medium. On the other hand, running high-density threshold simulations to the present age $(z=0)$ is computationally very challenging because of the extremely short timescales involved in the calculations. Only simulations performed with a low-density threshold are currently run to $z=0$ and fine-tuned to reproduce the observed low-redshift evolution of the star formation density.

We note that the mean-density region, because of its small dimensions, lacks massive haloes. In larger simulations, we expect to find rarer, larger, haloes, which can grow faster and host star formation in $\sim 10^{5} M_{\odot}-10^{6} M_{\odot}$ haloes.

We have performed high-resolution, three-dimensional, $\mathrm{N}$-body/SPH simulations including non-equilibrium atomic and molecular chemistry, star formation prescriptions, and feedback effects to investigate the onset of primordial star formation. We have studied how the primordial star formation rate changes according to different gas-density threshold, cosmological parameters, and simulation set-ups. Our main findings are summarized in the following:

- The typical low-density thresholds (below $\sim 1 \mathrm{~cm}^{-3}$ ) are inadequate for describing star formation episodes in mean regions of the universe at high redshift. To correctly estimate the onset of star formation, high-density thresholds are necessary.

- In rare, high-density peaks, the density can be higher than the usual low-density thresholds from very early times, therefore these prescriptions are not physically meaningful. Density thresholds lying beyond the isothermal peak (several particles per $\mathrm{cm}^{3}$, in our case) are still required: they should satisfy the Bate \& Burkert (1997) requirement $(N$ at least $\sim 2 N_{\text {neigh }}$ ) and Eq. (6) of Sect. 2. However, as long as they are beyond the isothermal peak, given the faster evolution in the phase diagram of the cooling particles in dense environments, the very exact value is not crucial.

- Different values of the threshold and the cosmological parameters can cause the onset of star formation at very different epochs: with a low-density threshold $\left(0.2 h^{2} \mathrm{~cm}^{-3}\right)$, star formation starts at $z \sim 25-31$ (depending on the cosmology), while high-density threshold models $\left(135 \mathrm{~h}^{2} \mathrm{~cm}^{-3}\right)$ predict a much later onset, at $z \sim 12-16$ (depending on the cosmology).

- Performing primordial, rare, high-density region simulations within the high-density threshold model, we find that the local star formation can set in as early as $z \sim 48$.

We conclude by adding a few comments on the meaning of the term "onset of star formation". From a purely physical point of view, the onset of star formation occurs when the proton-proton nuclear reactions ignite in a collapsed, dense core. Nowadays, in numerical simulations of cosmic structure evolution, this definition cannot be adopted, since it is not feasible to follow and resolve the behaviour of the gas on the very large range of scales involved. Therefore, the onset of star formation is meant as the attainment of a certain density threshold: once SPH particles reach it, they are assumed to be dense enough to collapse and to host star formation. This threshold can be viewed as a point of no return, because it imposes a limit above which the natural gas evolution is strongly altered by star production and feedback effects. The time when the threshold is reached and the time when the actual star formation takes place are typically not the same, but if the threshold is set appropriately, as discussed in the present work, they become very similar. Broadly speaking, one could consider assigning a simple prescription to standard numerical simulations based on the typical delay time of gas in fall. In this way, the onset of star formation could be easily corrected without implementing high-density thresholds or molecular evolution. In practice, this is not a trivial task: the time spent in the isothermal regime depends on numerous "environmental" factors, so different simulations with different initial conditions will be affected differently, according to their particular features. However, as an estimate, a typical free-fall time of $\sim 10^{2} \mathrm{Myr}$ is the one we expect in correspondence of $\sim 10^{-1} \mathrm{~cm}^{-3}$ (conventional low-density thresholds).

Throughout this paper, we have considered the onset of star formation to be the attainment of the density threshold, provided that the Jeans mass is resolved by a "large" number of SPH particles and the isothermal peak in the phase diagram is resolved (and we have seen in Sects. 2 and 4 that the latter two conditions are strongly related).

Acknowledgements. We acknowledge useful discussions with James Bolton, Massimo Ricotti, Cecilia Scannapieco, Volker Springel, Romain Teyssier, Michele Trenti, Simon D. M. White and John Wise. N.Y. thanks financial support from Grants-in-Aid for Young Scientists S from JSPS (20674003). We also acknowledge the anonymous referee for stimulating comments on the paper. The simulations were performed using the machines of the Max Planck Society computing center, Garching (Rechenzentrum-Garching) and of the MaxPlanck-Institut für Astrophysik. For the bibliografic research we have made use of the tools offered by the NASA Astrophysics Data System and by the JSTOR Archive.

\section{References}

Abel, T., Bryan, G. L., \& Norman, M. L. 2002, Science, 295, 93

Bate, M. R., \& Burkert, A. 1997, MNRAS, 288, 1060

Bate, M. R., Bonnell, I. A., \& Price, N. M. 1995, MNRAS, 277, 362

Bolton, J. S., \& Haehnelt, M. G. 2007, MNRAS, 382, 325

Brax, P. H., \& Martin, J. 1999, Phys. Lett. B, 468, 40

Bromm, V., \& Larson, R. B. 2004, ARA\&A, 42, 79

Bromm, V., Coppi, P. S., \& Larson, R. B. 2002, ApJ, 564, 23

Cen, R., \& Ostriker, J. P. 1992, ApJ, 399, L113

Ciardi, B., \& Ferrara, A. 2005, Space Sci. Rev., 116, 625

Crociani, D., Viel, M., Moscardini, L., Bartelmann, M., \& Meneghetti, M. 2008, MNRAS, 385, 728

Dalla Vecchia, C., \& Schaye, J. 2008, MNRAS, 387, 1431

Einstein, A. 1917, Sitzungsberichte der Königlich Preußischen Akademie der Wissenschaften, Berlin, 142

Evrard, A. E. 1988, MNRAS, 235, 911

Galli, D., \& Palla, F. 1998, A\&A, 335, 403

Gao, L., Yoshida, N., Abel, T., et al. 2007, MNRAS, 378, 449

Governato, F., Willman, B., Mayer, L., et al. 2007, MNRAS, 374, 1479

Gunn, J. E., \& Gott, J. R. I. 1972, ApJ, 176, 1

Hernquist, L., \& Katz, N. 1989, ApJS, 70, 419

Hinshaw, G., Weiland, J. L., Hill, R. S., et al. 2008, ArXiv e-prints, 803

Hollenbach, D., \& McKee, C. F. 1979, ApJS, 41, 555

Inutsuka, S.-I., \& Miyama, S. M. 1992, ApJ, 388, 392

Jeans, J. H. 1902, Phil. Trans., 199, A 1

Katz, N. 1992, ApJ, 391, 502

Katz, N., Weinberg, D. H., \& Hernquist, L. 1996, ApJS, 105, 19

Kennicutt, Jr., R. C. 1998, ARA\&A, 36, 189 
Larson, R. B. 1998, MNRAS, 301, 569

Maio, U., Dolag, K., Meneghetti, M., et al. 2006, MNRAS, 373, 869

Maio, U., Dolag, K., Ciardi, B., \& Tornatore, L. 2007, MNRAS, 379, 963

Maio, U., Ciardi, B., Dolag, K., \& Tornatore, L. 2008, in First Stars III, ed. B. W. O'Shea, \& A. Heger, AIP Conf. Ser., 990, 33

Nakamura, F., \& Umemura, M. 2001, ApJ, 548, 19

Navarro, J. F., \& White, S. D. M. 1993, MNRAS, 265, 271

Omukai, K., \& Palla, F. 2003, ApJ, 589, 677

Pawlik, A. H., Schaye, J., \& van Scherpenzeel, E. 2009, MNRAS, 394, 1812

Peebles, P. J. E. 1974, ApJ, 189, L51

Peebles, P. J. E., \& Dicke, R. H. 1968, ApJ, 154, 891

Peebles, P. J., \& Ratra, B. 2003, Rev. Mod. Phys., 75, 559

Ratra, B., \& Peebles, P. J. E. 1988, Phys. Rev. D, 37, 3406

Ricotti, M., Gnedin, N. Y., \& Shull, J. M. 2002a, ApJ, 575, 33

Ricotti, M., Gnedin, N. Y., \& Shull, J. M. 2002b, ApJ, 575, 49

Ricotti, M., Gnedin, N. Y., \& Shull, J. M. 2008, ArXiv e-prints, 802

Saitoh, T. R., Daisaka, H., Kokubo, E., et al. 2008, PASJ, 60, 667

Saslaw, W. C., \& Zipoy, D. 1967, Nature, 216, 976

Scannapieco, C., Tissera, P. B., White, S. D. M., \& Springel, V. 2005, MNRAS, 364,552
Schaye, J., \& Dalla Vecchia, C. 2008, MNRAS, 383, 1210

Schwarzschild, M., \& Spitzer, L. 1953, The Observatory, 73, 77

Springel, V. 2005, MNRAS, 364, 1105

Springel, V., \& Hernquist, L. 2003, MNRAS, 339, 289

Tegmark, M., Silk, J., Rees, M. J., et al. 1997, ApJ, 474, 1

Tornatore, L., Borgani, S., Dolag, K., \& Matteucci, F. 2007a, MNRAS, 382, 1050

Tornatore, L., Ferrara, A., \& Schneider, R. 2007b, MNRAS, 382, 945

Trenti, M., \& Stiavelli, M. 2009, ApJ, 694, 879

Truelove, J. K., Klein, R. I., McKee, C. F., et al. 1997, ApJ, 489, L179 Wetterich, C. 1988, Nucl. Phys. B, 302, 668

Whalen, D., O'Shea, B. W., Smidt, J., \& Norman, M. L. 2008, ApJ, 679, 925

White, S. D. M., \& Rees, M. J. 1978, MNRAS, 183, 341

Wiersma, R. P. C., Schaye, J., Theuns, T., Dalla Vecchia, C., \& Tornatore, L. 2009, ArXiv e-prints

Wise, J. H., \& Abel, T. 2007, ApJ, 665, 899

Wise, J. H., \& Abel, T. 2008, ApJ, 685, 40

Yoshida, N., Abel, T., Hernquist, L., \& Sugiyama, N. 2003, ApJ, 592, 645

Yoshida, N., Omukai, K., Hernquist, L., \& Abel, T. 2006, ApJ, 652, 6

Yoshida, N., Omukai, K., \& Hernquist, L. 2007, ApJ, 667, L117 